\title{
OSS 2010 Doctoral Consortium (OSS2010DC)
}

\author{
Walt Scacchi ${ }^{1}$, Kris Ven ${ }^{2}$, and Jan Verelst ${ }^{2}$ \\ 1 University of California, Irvine, USA \\ 2 University of Antwerp, Belgium \\ http: / / www . ua.ac.be/oss2010dc
}

\section{Goal}

The goal of the Doctoral Consortium is to provide $\mathrm{PhD}$ students with an environment in which they can share and discuss their goals, methods and results before completing their research. Participants will be selected based on the quality of the proposed research, its potential significance and contribution to the OSS domain, and the potential benefit of the Doctoral Consortium to the PhD student's research.

The Doctoral Consortium will take place on May 30, allowing participants to attend the OSS 2010 conference after the Doctoral Consortium. This allows PhD students to further discuss their research with other researchers in the following days.

As well, because of the diversity of the communities involved, the Doctoral Consortium will allow PhD students to make connections beyond their own disciplines. As a result, we expect that participation will allow $\mathrm{PhD}$ students to develop a better understanding of the different research communities, which we believe will facilitate their participation in future inter-disciplinary research.

We will also invite other faculty members to attend the Doctoral Consortium to stimulate discussion.

\section{Scope}

The scope of research topics of the Doctoral Consortium is the same as for the main conference. We therefore invite submissions related to all aspects of open source software including, but not limited to software engineering perspectives, emerging perspectives, social science, and studies of OSS deployment.

We invite submissions from PhD students in the early stages of their research (e.g., those who are at the end of their first year or in their second year), as well as in the late stages of their research (e.g., those who are close to graduating).

PhD students who apply for the Doctoral Consortium should at least have decided on a research topic or topic area, and have a proposal for an appropriate research method. Preferably, $\mathrm{PhD}$ students should still have the time to incorporate the feedback obtained during the Doctoral Consortium in their dissertation.

\section{Full Papers}

All full papers submitted to the Doctoral Consortium will be peer reviewed by at least two independent reviewers. PhD students that are accepted to the Doctoral Consortium, 
will give a presentation of their work. We aim to provide sufficient time for discussion (at least 20 minutes) to ensure that $\mathrm{PhD}$ students obtain quality feedback from the Doctoral Consortium co-chairs, the members of the program committee, as well as other PhD students. This feedback will allow them to enhance their own research proposal. Subsequently, doctoral students whose advisory committee lacks sufficient expertise with current OSS research may benefit in a number of ways from participating in the Doctoral Consortium with attending faculty.

\section{Lightning Talks}

Similar to last year, we will be hosting a special session of "lightning talks" during the OSS 2010 Doctoral Consortium. During this lightning talks session, multiple PhD students will be able to briefly present their research proposal. Each presenter will be provided with a 3-minute time slot and will have one slide available. The lightning talks session allows PhD students to give a brief presentation of their research, to actively participate in the Doctoral Consortium, and to generate awareness of their topic.

The lightning talks session is primarily targeted towards $\mathrm{PhD}$ students who are in the early phases of their research. Attending the discussion on the research proposals of other PhD students may also be beneficial for them, as it provides ideas on what future reactions to their own research may be. In addition, by giving a lightning talk, they are able to generate an interest in their research topic, which allows them to connect to other researchers in related areas and to gain preliminary feedback on their proposal.

\section{Doctoral Consortium Chairs}

Walt Scacchi

Kris Ven

Jan Verelst

\section{Program Committee}

Kevin Crowston
Joseph Feller
Daniel M. German
Jesus Gonzalez-Barahona
Björn Lundell
Maha Shaikh

University of California, Irvine

USA

University of Antwerp

University of Antwerp
Belgium

Belgium
Syracuse University USA

University College Cork Ireland

University of Victoria Canada

Universidad Rey Juan Carlos Spain

University of Skövde Sweden

London School of Economics UK 